Journal of Oral Science, Vol. 44, No. 3/4, 161-164, 2002

Case report

\title{
An alternative method for fabricating a closed hollow obturator: a clinical report
}

\author{
Hüseyin Yazicioğlu and Suat Yaluğ \\ Department of Prosthodontics, Faculty of Dentistry, Gazi University, Ankara, Turkey
}

(Received 4 June and accepted 17 September 2002)

\begin{abstract}
This article describes a procedure in which the fabrication of a closed hollow obturator can be made with three sections of a denture flask by using silicone. Ease of fabrication while controlling the thickness of the hollow portion and eliminating leakage and discoloration are major advantages of this technique while minimizing laboratory and clinical appointment time. (J. Oral Sci, 44, 161-164, 2002)
\end{abstract}

Key words: obturators; closed hollow obturator; maxillofacial prosthetic; maxillectomy.

\section{Introduction}

Maxillectomy results in communication of the oral and nasal cavities, leading to dysfunctions in speech, mastication and swallowing. Placement of an obturator prosthesis can re-establish oronasal separation and improve these oral functions $(1,2)$.

Since the sixteenth century, maxillary surgical defects have been restored by obturator prosthesis made from a variety of materials and techniques (3). There are numerous references in the literature that describe various methods for fabricating open and closed hollow obturator prosthesis (4-7). Both types of obturators allow for the fabrication of a lightweight prosthesis that is readily tolerated by the patient, while effectively extending into the defect (4).

If the obturator is left open, nasal secretions accumulate leading to odour and added weight. If secretions do tend to accumulate, a small diagonal opening may be

Correspondence to Dr. Hüseyin Yazicioğlu, Department of Prosthodontics, Faculty of Dentistry, Gazi University, Ankara, Turkey

Tel: (90)312-2126220

Fax: (90)312-2239226

E-mail: hyazici@gazi.edu.tr made between the inferior-lateral floors of the obturator through the cheek surface for drainage. The other disadvantages of an open type hollow obturator include difficulty in polishing and cleaning the internal surface, from saliva, mucous crusts, food accumulation, and inability to obtain support from the superior aspect in the defect area (8).

Closed obturators have the advantage of eliminating the pooling of moisture while extending superiorly into the defect and the reducing air space. Often, patients are presented with the need for an extraoral prosthesis in addition to an intraoral prosthesis. The closed hollow obturator benefits these patients by allowing the attachment of intraoral and extraoral prosthesis to each other, thus providing additional retention and stability (6).

To decrease the weight of the obturator prosthesis several techniques have been proposed for the fabrication of a hollow acrylic resin obturator. Among the methods suggested are the method of fabricating the bulb part of the prostheses then connecting these two parts in the mouth with autopolymerized acrylic resin (9); after the replacement of sugar or ice in the defect region during acrylic polymerization, removing the ice or sugar from the processed prostheses with the help of a hole made, then closing this region with autopolymerized acrylic resin (10). Another technique is to coat the defect region with two plates of wax, then asbestos is placed in this region. The wax and asbestos are taken out. During packing procedure of heat polymerized acrylic resin, asbestos is placed once more. And when the packing is over they are removed again and the flasking procedure is completed (5).

\section{Clinical summary}

A 55-year-old man who underwent a maxillectomy for adenoid cystic carcinoma arising in the hard palate served as a patient for this study. After the surgery, in order to hold the surgical dressings, close the area of resection 
and provide limited physiologic assistance for speech and deglutition, a surgical obturator prosthesis was placed. Five months after the surgery, fabrication of a definitive obturator prosthesis was planned to restore deglutition and speech after confirmation of nonreccurence of the tumor and no change in tissue during healing. Oral examination revealed that the surgically acquired defect involved the hard palate and nasal cavity (Fig. 1).

\section{Procedure}

A preliminary impression was made to produce a cast of the maxillary defect on which a custom tray was fabricated.

1. An impression of the defect was then taken with alginate (Algiplast HP, Tissi Dental, Italy) on the custom tray to construct the master cast. After undesirable undercuts within the defect were blocked out on the master cast by using wax (Polywax, Turkiye) and autopolymerized acrylic resin record base, the wax occlusion rim was fabricated.

2. The maxillo-mandibular relations were recorded. The

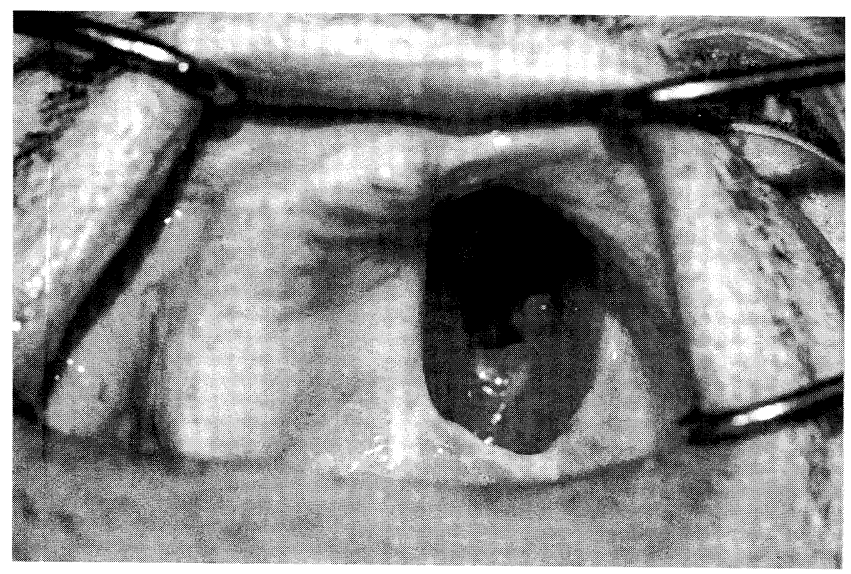

Fig. 1 Occlusal view of maxillectomy defect.

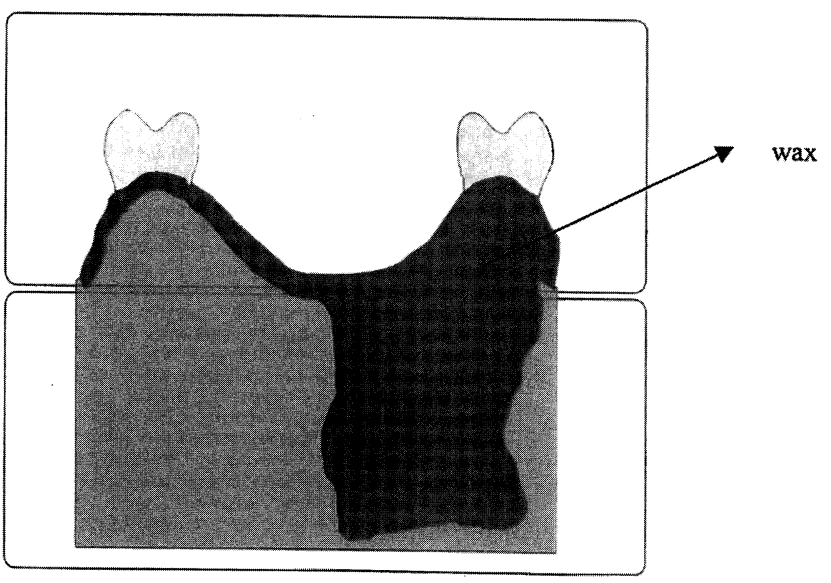

Fig. 2 The obturator placed in the flask. teeth were set in wax and clinically verified with a wax try-in. The waxing was finished and sealed to the cast for investing, then the wax was boiled out (Fig. 2). After the wax was eliminated, the flask was opened and the undercuts were blocked out along the floor. The walls of the defect area were sealed with wax to a minimal thickness of $3 \mathrm{~mm}$.

3. Another top flask was placed on the waxing of the hollow defect. The entire surface of the inner flask was invested with silicone of $0.5 \mathrm{~cm}$ thickness (Polysiloxane-condensation curing, Durosil L, President, Germany) (Fig. 3) and in order to maintain the retention between the silicone and plaster, retentive areas were created. After the silicone condensed, the remaining part of the flask was invested with plas-

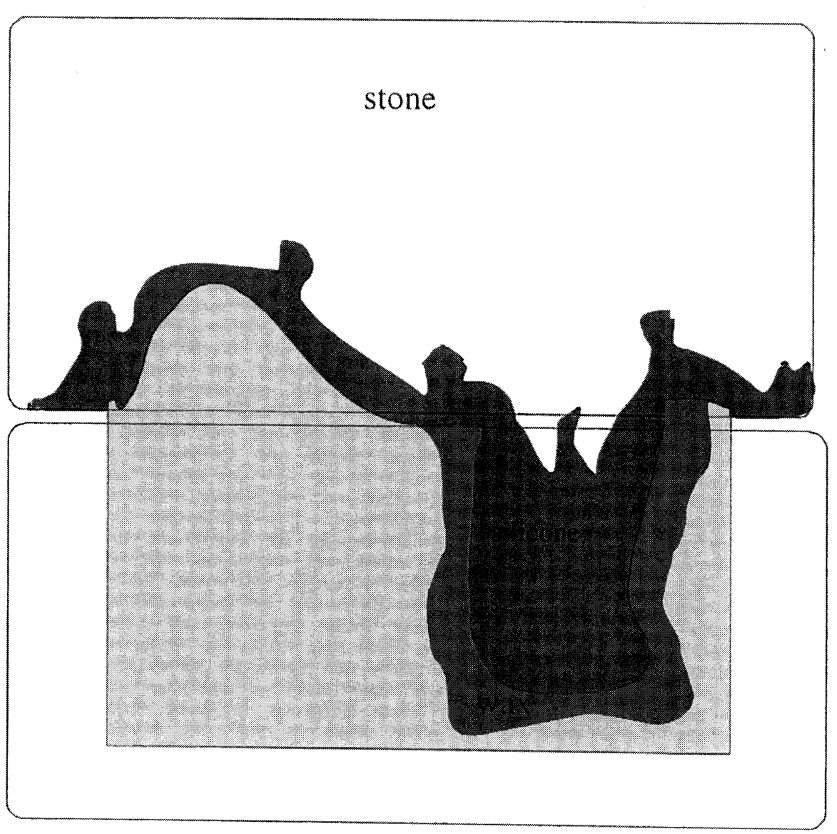

Fig. 3 Defect area is plated with $3 \mathrm{~mm}$ wax and the flask is coated with $0.5 \mathrm{~cm}$ silicone.

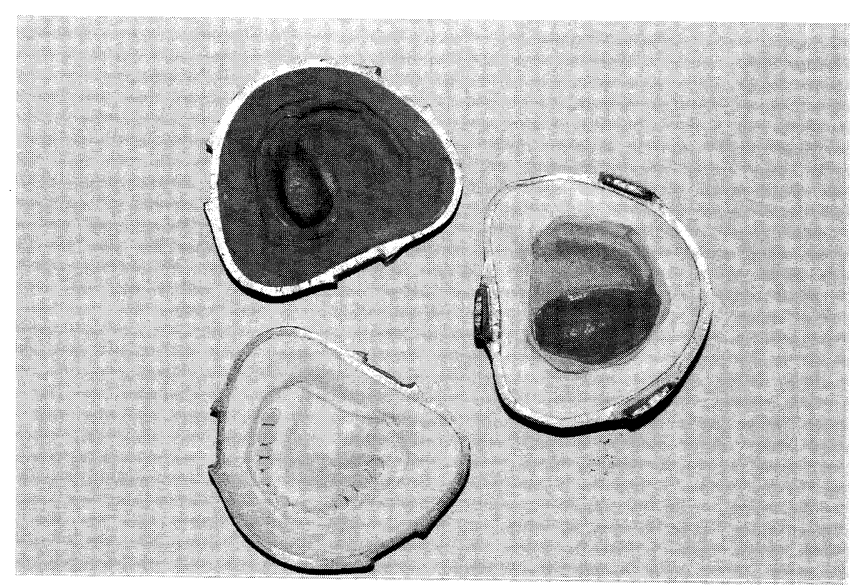

Fig. 4 Appearance of flasks. 
ter. The flask was opened after the plaster was set (Fig. 4). The wax was boiled out. The hollow section was packed and processed with heat-polymerized acrylic resin (Vertex RS, Dentimex, Holland).

4. The flask was separated and the silicone was gently removed from the obturator section. In order to maintain the continuity of the heat-polymerized acrylic resin that was to be packed on the initial flask, $1.5 \mathrm{~mm}$ of wax was placed on the other part of the resin region to create a space after the boiling out process. Wax with a thickness of $1.5 \mathrm{~mm}$ was applied evenly on the initial flask with the invested teeth and autopolymerized acrylic resin with a thickness of $1.5 \mathrm{~mm}$ (Vertex Self-Curing, Dentimex, Holland) was applied on wax (Fig. 5). The flask was completely seated. After the autopolymerized acrylic resin had set, the flasks were separated (Fig. 6).

5. The wax was removed and all areas of the processed base were roughened, then the heat polymerized

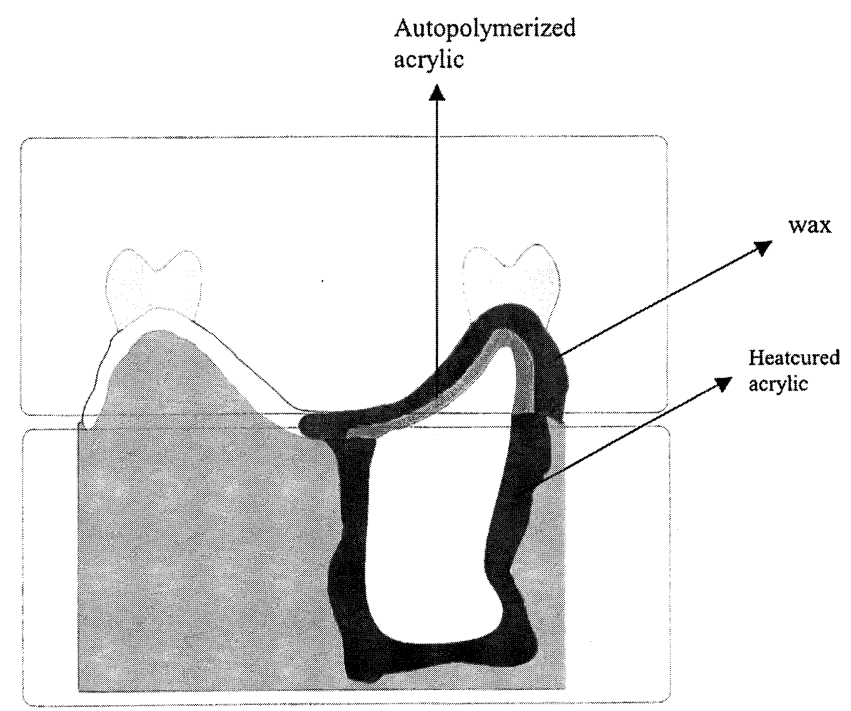

Fig. 5 Autopolymerized acrylic is placed.

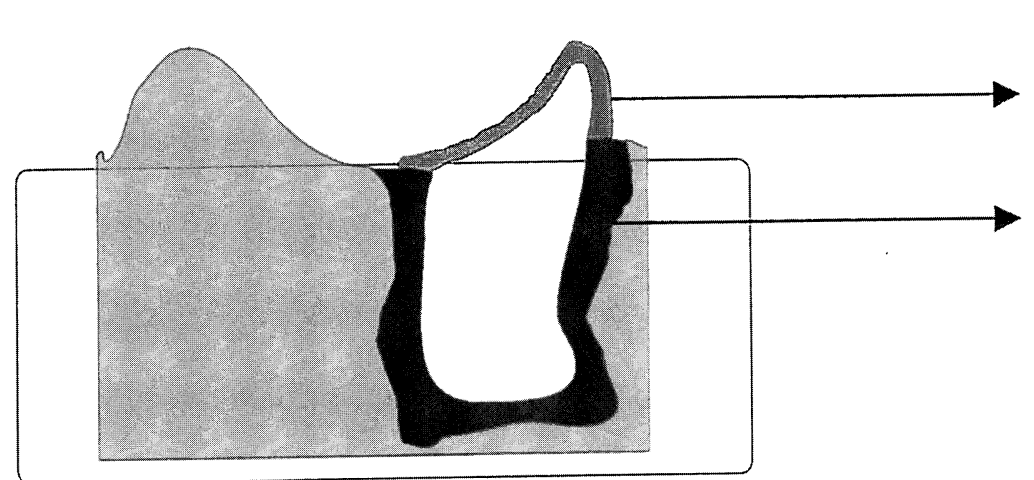

Autopolymerized

acrylic

Heatcured

acrylic

Fig. 6 Fabricating of hollow-bulb. 


\section{Discussion}

The method for fabricating this hollow obturator was modified from a technique described by McAndrew et al. (4). This technique allows for control of the wall thickness of the obturator extension, thereby, minimizing the weight of the prosthesis. Further all surfaces exposed to the oral cavity are processed with heat-polymerized acrylic resin. This technique eliminates an additional step that is described by Minsley et al. (7).

In this technique, a thickness of $3 \mathrm{~mm}$ was obtained in all surfaces of defect and tooth area and a uniform space was maintained at the area of defect by using an initial flask of the obturator. In contrast, $3 \mathrm{~mm}$ of acrylic thickness in the defect area was obtained with the uncontrolled acrylic thickness in the tooth area in McAndrew's technique because an initial flask was not used, creating a random space in front of the tooth area. We used silicone in the second flask whereas McAndrew used stone. Because silicone was used in our technique, no locking was seen on both the acrylic of the defect region and the surface of impression. Among the bulb fabricating methods, with the sugar and ice technique (10), the hole made for removing the ice and sugar is closed with autopolymerized acrylic resin and because of this, leakage and colorization occur (4). In our method there was no relation between the autopolymerized acrylic resin and oral cavity. It is thought that there will be no leakage to the hollow bulb and no colorization in heat cured acrylic resin used as base materi$\mathrm{al}$, and this is one of the advantages of this method besides the other one reported above. On the other hand, the trouble of cleaning the undefected regions plaster from the defect is eliminated.

\section{References}

1. Kanazawa T, Yoshida H, Furuya Y, Shimodaira K (2000) Sectional prosthesis with hollow obturator portion made of thin silicone layer over resin frame. J Oral Rehabil 27, 760-764

2. Shimodaira K, Yoshida H, Mizukami M, Funakubo $\mathrm{T}$ (1994) Obturator prosthesis confirming to movement of the soft palate: A clinical report. J Prosthet Dent 71, 547-551

3. DaBreo EL (1991) Dimensional change in maxillary prosthetic obturators. J Prosthet Dent 66, 669-673

4. McAndrew KS, Rothenberger S, Minsley GE (1998) 1997 Judson C. Hickey Scientific Writing Awards. An innovative investment method for the fabrication of a closed hollow obturator prosthesis. $\mathbf{J}$ Prosthet Dent 80, 129-132

5. Worley JL, Kniejski ME (1983) A method for controlling the thickness of hollow obturator prosthesis. J Prosthet Dent 50, 227-229

6. Shifman A (1983) A technique for the fabrication of the open obturator. J Prosthet Dent 50, 384-385

7. Minsley GE, Nelson DR, Rothenberger SL (1986) An alternative method for fabrication of a closed hollow obturator. J Prosthet Dent 55, 485-490

8. Keyf F (2001) Obturator prostheses for hemimaxillectomy patients. J Oral Rehabil 28, 821-829

9. Brown KE (1969) Fabrication of a hollow-bulb obturator. J Prosthet Dent 21, 97-103

10. Schneider A (1978) A method of fabricating a hollow obturator. J Prosthet Dent 40, 351 